\title{
Spin-Based Quantum Dot Quantum Computing
}

\author{
Xuedong $\mathrm{Hu}$ \\ Department of Physics, University at Buffalo, The State University of New York, \\ 239 Fronczak Hall, Buffalo, NY 14260-1500, USA xhu@buffalo.edu
}

\section{Introduction}

During the past decade, the study of quantum computing and quantum information processing has generated wide spread interest among physicists from areas ranging from atomic physics, optics, to various branches of condensed matter physics 1, 2. The key thrust behind the rush toward a working quantum computer (QC) was a quantum algorithm designed by a computer scientist, Peter Shor from AT\&T, that can factor large numbers exponentially faster than any available classical algorithms 3 . This exponential speedup is due to the intrinsic parallelism in the superposition principle and unitary evolution of quantum mechanics, thus it requires a computer that is made up of quantum mechanical parts (qubits), whose evolution is governed by quantum mechanics. Since the invention of Shor's factoring algorithm, it has also been proved that error correction can be done to a quantum system [4, so that a practical QC does not have to be forever perfect to be useful. After these two important developments, the field of quantum computation has seen an explosive growth.

Many physical systems have been proposed as candidates for qubits in a QC. Prominent examples include trapped ions [5, 6], photons or atoms in cavities 7, 8, nuclear spins in a liquid NMR system 9, electron spin in semiconductor quantum dots 10, 11, donor electron or nuclear spins in semiconductors [12, 13, 14], structures consisting of superconducting Josephson junctions [15, and many more. The experimental demonstration of quantum coherence and maneuverability of these physical systems can be characterized as very fruitful in some cases, such as trapped ions [16] and liquid state NMR [17, to preliminary in many other cases, such as most solid state schemes.

Although experimental progress in many solid state schemes has been slow to come, they are still often considered promising in the long term because of their perceived scalability. After all, the present computer technology is based on semiconductor integrated circuits with ever smaller feature size. However, it is still to be demonstrated whether and how the available semiconductor technology can help scale up an architecture that is quantum mechanically coherent.

Here we first present a brief overview of the current theoretical and experimental progresses in the study of quantum dot-based quantum computing 
schemes. We then focus on the spin-based varieties, which are generally regarded as the most scalable because of the relatively long coherence times of electron and nuclear spins.

\section{General Features of the Quantum Dot Quantum Computing Schemes}

\subsection{Classification of the QC Schemes}

Many proposals have been made to use quantum dot (QD) and various electron and nuclear degrees of freedom to process quantum information. Crudely these proposals can be classified as charge-based and spin-based (both electron and nuclear spins). We comment on a few of the charged-based proposals below and then discuss in more detail the particular spin-based schemes on which we focus in this lecture.

One of the earliest charge-based proposal is to use the lowest two orbital energy levels of a single electron QD [18. When an external electric field is applied to the QD, the ground and first excited states would acquire opposite electric dipole moments (the so-called quantum-confined Stark effect [19]). With the interdot tunneling completely suppressed, the inter-qubit coupling is then dominated by dipolar interaction. It creates energy shifts in the energy levels of one QD depending on the state of its neighboring dot, thus provides the physical basis for conditional two-qubit operations [18, 20. Resonant optical pulses can then be used to implement the conditional excitations.

Several charge-based proposals have since been suggested with more concrete architectures and more detailed physical descriptions. Examples include those using pillars of vertically stacked QDs 21, and chains of horizontal double dot 22, 23. Similar dipole-interaction-based proposals were also put forward in other physical systems such as trapped neutral atoms [24]. Alternatively, there have also been suggestions using cavity modes (instead of dipole interaction) to couple electronic orbital states [25]. Indeed, the prominent charge-based qubit is the Cooper pair box proposal in superconductor, where the charged and uncharged state of a small superconducting grain form the basis of a qubit [15, 26, 27, 28, 29. The semiconductor analogue of the coherent charge oscillation experiment [27] has just recently been done [30, although with much shorter coherent time compared to the superconducting counterpart. The great experimental successes in Cooper pair boxes have also prompted searches for other systems that share some common features with Cooper pair boxes. Examples include such exotic systems like quantum Hall bilayers [31, 32.

All the charge-based schemes mentioned so far use singly charged semiconductor QDs. The associated strong Coulomb interaction provide a convenient means for fast qubit manipulation, but can also lead to fast decoherence. One way to alleviate this problem is to use neutral excitations such as excitons 
as qubits, where there is the added benefit that excitons can be precisely controlled optically. Indeed, uncharged QDs have been proposed as possible candidates for quantum information processing [33, 34, 35, 36, 37, and many experiments have been done to demonstrate exciton coherence and control in a single QD 38, 39, 40, 41, 42, 43. Here single excitons are optically excited in individual QDs and can be coherently manipulated optically. The presence and absence of an exciton in a QD provide the two states of a qubit. Again, entanglement between different qubits is based on Coulomb renormalization of the energy levels. The exciton-based QC proposals clearly illustrate the dichotomy faced by all $\mathrm{QC}$ architectures: excitons are neutral, therefore are more insulated from their environment and decohere more slowly than the single charge based schemes. However, the charge neutrality also strongly reduces the interaction between spatially separated excitons, thus rendering it more difficult to perform entangling operations.

In the following we will focus on spin-based QD QC architectures. A fermionic spin, more specifically a spin- $1 / 2$, being a quantum two-level system in a finite magnetic field, is a natural qubit with its spin-up and spindown states. For example, the most successful experimental demonstration of quantum control and entanglement is in a trapped ion system using two hyperfine split nuclear spin levels for qubit [5]. It was discussed in the mid1990s that Ising interaction between electron spins in solids can produce the desired entanglement for a QC [44. Here, the specific common thread among the schemes we will concentrate on is that in all of them direct electron exchange coupling plays a crucial role. There are certainly proposals where electron spin interaction takes other forms (such as cavity photon mediated [11, free electron mediated [13, 45], optical RKKY interaction [46], and dipole coupling [47]), and there have been a tremendous amount of research done on the optical characterization of electron and nuclear spins 48, 49, 50, 51. Nevertheless, we are going to be mostly focused on the electrical control of spins and their interaction.

\subsection{GaAs Quantum Dot QC Architecture}

One of the earliest proposed solid state QC schemes uses the spin of a single electron trapped in a GaAs QD as its qubit (see [52, 10, 53, 54, 55] and references therein, and Fig. (1). Local magnetic fields are used to manipulate single spins, in the sense that it creates a local Zeeman splitting, which can then be accessed by a resonant RF pulse. Inter-dot exchange interaction, which is a purely spin interaction in the form $J \mathbf{S}_{1} \cdot \mathbf{S}_{2}$ but of electrostatic origin, is used to couple neighboring spins and introduce two-qubit entanglement. A single trapped electron in a GaAs QD ground orbital state means very low spin-orbit coupling as the electrons occupy states at the bottom of the GaAs conduction band and have essentially $\mathrm{S}$ type states [55]. Thus the electron spin coherence time should be even longer than in the bulk, where electron spin decoherence is already very slow. 


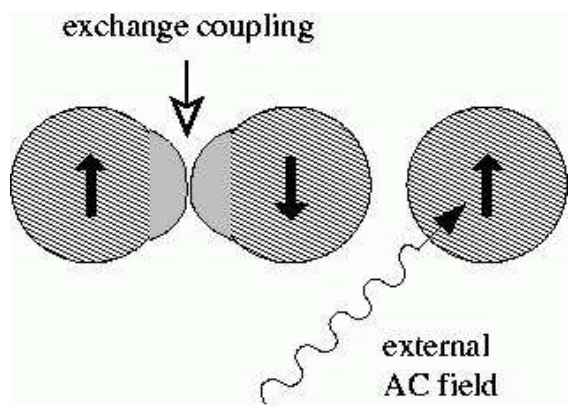

Fig. 1. A schematic of a QD QC.

Some of the critical issues regarding GaAs QD QC are trapping a single electron in a gated $\mathrm{QD}$, producing a local electron Zeeman splitting that is different from all its neighbors (so that resonant single spin rotations can be performed), creating and controlling a finite exchange coupling between electrons in neighboring QDs, and last but not least, measuring the single electron spins with high fidelity.

\subsection{Si Quantum Dot QC Architecture}

The GaAs QD QC architecture we discussed above can be relatively easily extended to a $\mathrm{Si} / \mathrm{SiGe}$ material system [56]. Here the electrons are confined in the pure (but strained) silicon region (instead of SiGe alloys), while the confinement is produced by the SiGe alloys along the growth direction and surface gates in the in-plane directions (Fig. 11). Compared to GaAs, electron spin decoherence due to hyperfine coupling (as we will discuss in Section 3) in such a system can be suppressed by using purified ${ }^{28} \mathrm{Si}$ as the host material. On the other hand, the more complicated band structure of silicon and the less well-controlled interface may pose problems to the coherent manipulation of a quantum device, which needs to be further studied.

\subsection{Si Donor Nuclear Spin QC Architecture}

The QDs underlying the previous two QC schemes are essentially artificial atoms, where electron confinement is provided by the barrier materials and the external electrostatic potential from the metallic gates on the surface of the devices. A naturally occurring alternative is the weakly bound donor states. Take an example of a monovalent donor, where one extra proton is present at the donor nuclear site while an extra donor electron is loosely bound to this proton. Now the donor location becomes the tag for the bound electron spin or the resident nuclear spin, and identical copies of such donors can be easily made in a semiconductor. 
One of the most intriguing and influential QC schemes is the donor nuclear spin based Si QC [12], as is shown in Fig. 2(a). Here spin- $1 / 2{ }^{31} \mathrm{P}$ donor nuclei are qubits, while donor electrons together with external gates provide single-qubit (using external magnetic field) and two-qubit operations (using hyperfine and electron exchange interactions). Specifically, the single donor nuclear spin splitting is given by 12 .

$$
\hbar \omega_{A}=2 g_{n} \mu_{n} B+2 A+\frac{2 A^{2}}{\mu_{B} B}
$$

where $g_{n}$ is the nuclear spin g-factor $\left(1.13\right.$ for $\left.{ }^{31} \mathrm{P}[12]\right), \mu_{n}$ is the nuclear magneton, $A$ is the strength of the hyperfine coupling between the ${ }^{31} \mathrm{P}$ nucleus and the donor electron spin, and $B$ is the applied magnetic field. It's clear that by changing $A$ one can effectively change the nuclear spin splitting, thus allow resonant manipulations of individual nuclear spins. If the donor electrons of two nearby donors are allowed to overlap, the interaction part of the spin Hamiltonian for the two electrons and the two nuclei include electron-nuclear hyperfine coupling and electron-electron exchange coupling 12]:

$$
\begin{aligned}
H & =H_{\text {Zeeman }}+H_{\text {int }} \\
& =H_{\text {Zeeman }}+A_{1} \mathbf{S}_{1} \cdot \mathbf{I}_{1}+\mathbf{A}_{2} \mathbf{S}_{2} \cdot \mathbf{I}_{2}+J \mathbf{S}_{1} \cdot \mathbf{S}_{2},
\end{aligned}
$$

where $\mathbf{S}_{1}$ and $\mathbf{S}_{2}$ represent the two electron spins, $\mathbf{I}_{1}$ and $\mathbf{I}_{2}$ represent the two nuclear spins, $A_{1}$ and $A_{2}$ are the hyperfine coupling strength at the two donor sites, and $J$ is the exchange coupling strength between the two donor electrons, which is determined by the overlap of the donor electron wavefunctions. The lowest order perturbation calculation (assuming $A_{1}=$ $A_{2}=A$ and $J$ is much smaller than the electron Zeeman splitting) results in an effective exchange coupling between the two nuclei and the coupling strength is

$$
J_{n n}=\frac{4 A^{2} J}{\mu_{B} B\left(\mu_{B} B-2 J\right)} .
$$

Now the two donor electrons are essentially shuttles between different nuclear spin qubits and are controlled by external gate voltages. The final measurement is done by first transferring nuclear spin information into electron spins using hyperfine interaction, then converting electron spin information into charge states such as charge locations [57]. A significant advantage of silicon is that its most abundant isotope ${ }^{28} \mathrm{Si}$ is spinless, thus providing a "quiet" environment for the donor nuclear spin qubits. In addition, Si also has smaller intrinsic spin-orbit coupling than other popular semiconductors such as GaAs. In general, nuclear spins have very long coherence times because they do not strongly couple with their environment, and are thus good candidates for qubits. However, this isolation from the environment also brings with it the baggage that individual nuclear spins are difficult to control and measure. 
This is why donor electrons play a crucial role in the Si QC scheme. On top of the good material properties Si possesses, there is another potential advantage of a QC based on Si: the prospect of using the vast resources available from the Si-based semiconductor chip industry. In Bruce Kane's own words, it is always advantageous if one can have the eight hundred pound gorilla on his/her side.

(a) Donor nuclear spin Si quantum computer

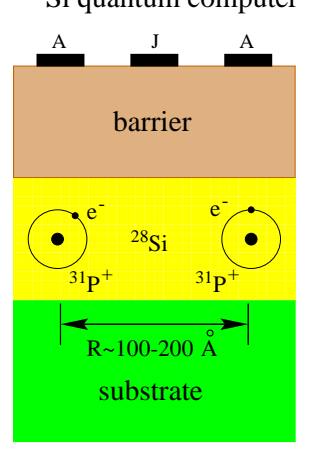

(b) Donor electron spin $\mathrm{SiGe}$ quantum computer

$\mathrm{z}_{4}$

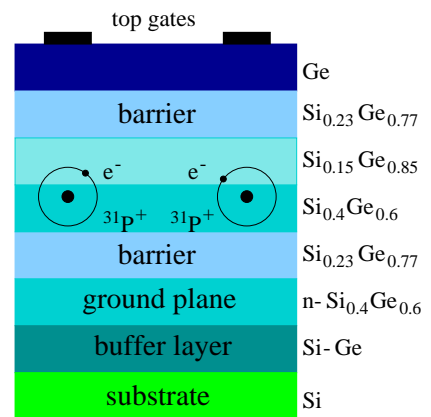

Fig. 2. Schematics of (a) a Si donor nuclear spin QC and (b) a Si donor electron spin QC.

\subsection{Si Donor Electron Spin QC Architecture}

A direct Si donor electron analogue to the GaAs QD QC scheme has also been proposed [14. In this scheme the ${ }^{31} \mathrm{P}$ donor electron spins are employed as qubits (Fig. 2(b)). The phosphorus donors are located in a Si layer sandwiched in between SiGe alloy layers. By moving a donor electron into alloy regions of a different $\mathrm{g}$-factor, its Zeeman splitting can be tuned significantly, which then allows selective single-qubit operations using resonant RF pulses. Similarly, different alloy regions also present different electron effective masses, which affect the size of the donor electron wavefunction sensitively. Such property can then be used to tune the exchange coupling between two bound donor electrons, and two-qubit operations are again provided by the direct exchange interaction between neighboring donor electrons. This all-electron proposal has much faster gate operations compared to the nuclear-electron hybrid scheme mentioned above. However, the alloy environment has to be more thoroughly studied for such a QC scheme to be considered more practical. 


\section{Electron Spin Coherence in Semiconductors}

To use spins as qubit for a QC, they should possess very long coherence time. Here "long" is in the sense that within a characteristic spin coherence time a large number of operations can be performed. The criteria for the large number is determined by the requirements of successfully performing quantum error correction, which lead to numbers in the range of $10^{4}$ to $10^{6}$ [1. For example, if a gate operation on an electron spin can be performed in $1 \mathrm{~ns}$, then its coherence time needs to be longer than $10 \mu \mathrm{s}$.

Spin coherence (whether it is electron spin or nuclear spin) is regularly described in terms of a longitudinal (or spin-lattice) relaxation time $T_{1}$ and a spin-spin relaxation time $T_{2}$ [58. These descriptions originate from the magnetic resonance studies of these spin species going back to the 1940s [59, 60. $T_{1}$ generally describes processes that involve energy transfer between a spin and its environment, while $T_{2}$ describes everything that disrupts the quantum coherence of a spin, thus is generally much shorter than $T_{1}$.

Notice that spin-flip processes cause both population relaxation and dephasing, contributing to both rates $1 / T_{1}$ and $1 / T_{2}$. However, in a real physical system the longitudinal and transverse directions are often affected differently by the environment. Indeed, there exist pure dephasing processes that affect only $T_{2}$ but not $T_{1}$. One example is the colliding molecules (which can be described as a pseudospin system, with spin up and down referring to the two relevant internal levels of the molecules) in an optically active gaseous medium, where molecules constantly undergo collisions with each other, some of them inelastic, but most of them elastic. During an inelastic collision, electrons in the molecules undergo transitions that correspond roughly to spin relaxation (or complete loss of the system as an electron gets excited out of the two optically active levels). During an elastic collision, the single molecule energy spectrum changes due to the presence of the other molecule nearby. This shift in energy levels (particularly the two active levels) is dependent on the details of the collision, and is thus a random variable, which we refer to as $\delta \omega(t)$. Including this frequency shift, the differential equation for the off-diagonal density matrix element $\rho_{\uparrow \downarrow}$ (representing the coherence between the up and down levels) becomes [61]

$$
\dot{\rho}_{\uparrow \downarrow}(t)=-i[\omega+\delta \omega(t)] \rho_{\uparrow \downarrow} .
$$

Note that in the first order approximation the population in the up level, $\rho_{\uparrow \uparrow}$, is not affected by this level splitting fluctuation since its equation of motion is independent of the energy difference $\omega$ [61] (in other words, the fluctuation in the level splitting does not lead to population relaxation). $\delta \omega(t)$ is a random variable that averages to zero, $\langle\delta \omega(t)\rangle=0$. Within the Markovian approximation, the random fluctuation in energy level splitting of the two level system causes a pure dephasing effect:

$$
\left\langle\rho_{\uparrow \downarrow}(t)\right\rangle=\left\langle\rho_{\uparrow \downarrow}(0)\right\rangle e^{-i \omega t} e^{-\gamma_{p h} t} .
$$


This pure dephasing only contributes to $T_{2}$ of a spin or pseudospin system, but not to $T_{1}$.

Another well-known example of pure dephasing is the dipolar spin-spin interaction between nuclear spins in a solid, which produces effective local magnetic field fluctuations for each nuclear spin and hence contributes essentially only to $T_{2}$ (the corresponding effect on $T_{1}$ is extremely small). What is important for dephasing is that some change in the state of the environment must occur due to its interaction with the system - dephasing does not require an inelastic scattering process in the system, although all inelastic scatterings necessarily produce dephasing. In fact, as mentioned before, $T_{2}$ in the context of electron spin resonance (ESR) and nuclear magnetic resonance (NMR) is often called the spin-spin relaxation time because the most important intrinsic effect contributing to $T_{2}$ is the dipolar interaction among various spins in the system, which, while transferring energy among the spins themselves, does not lead to overall energy relaxation from the total spin system (and does not change the total magnetic moment). By contrast, spin-lattice interactions lead to energy relaxation (via spin-flip processes) from the spin system

to the lattice, and thus contribute to $T_{1}^{-1}$, the spin-lattice relaxation rate. In short, $T_{2}$ sets the time scale for the spin system to achieve equilibrium within itself whereas $T_{1}$ sets the time scale for the global thermodynamic equilibrium between the spin system and the lattice. For the purpose of quantum computing, it is obvious that $T_{2}$ is the directly relevant time scale, because we need to keep all the spins completely quantum coherent.

As we mentioned before, it is imperative that $T_{2}$ for an electron spin in a single QD is a factor of $10^{4}$ or so greater than the typical gating time in a QD QC 2] in order for the quantum computing process to be fault tolerant. For $B=1 \mathrm{~T}$, the Zeeman splitting in a GaAs QD is about $0.03 \mathrm{meV}$, which yields 100 ps for the precession time of one spin, which can be used as the one qubit gate (the two qubit gate time is shorter, $\hbar / J \sim 50$ ps for $J \sim 0.1$ $\mathrm{meV}$ ). Therefore for quantum error correction to be performed reliably, $T_{2}$ for the trapped electron spin needs to be on the $\mu$ s time scale, which may very well be the case at low enough temperatures in a single QD. We note that the existing experimental estimates of free electron spin relaxation time $T_{2}$ in GaAs (for $T=1-4 \mathrm{~K}$ ) is around 10-100 ns 69, which is obviously a lower bound.

\subsection{Spin Decoherence Channels in Semiconductors}

In doped semiconductors there are three major spin relaxation mechanisms: Elliot-Yafet (EY), Dyakonov-Perel' (DP), and Bir-Aronov-Pikus (BAP) mechanisms, for conduction electrons [48, 62] at not-too-low temperatures. The origin of the EY mechanism is spin-orbit coupling. Spin-orbit coupling does not lead to spin relaxation by itself, but it mixes the electron orbital and spin degrees of freedom. When combined with another scattering mechanism, such as phonon emission/absorption and impurity scattering, electron spin-flip can 
occur (spin-flip now means the dominant spin component in the Bloch state changes). In the DP mechanism, the splitting of spin up and down conduction bands due to lack of inversion symmetry (as in III-V semiconductors such as GaAs, which has the zinc-blende lattice structure) acts as an effective momentum dependent magnetic field $\mathbf{B}(\mathbf{k})$. An electron with momentum $\mathbf{k}$ and spin $\mathbf{S}$ precesses in this effective field $\mathbf{B}(\mathbf{k})$ and loses its spin memory. As this electron is scattered into a different $\mathbf{k}$ state, its spin will start to precess around the new effective field. This constant change of effective magnetic field actually reduces the electron spin relaxation, so that the spin relaxation time is inversely proportional to the momentum relaxation time in this mechanism (cf motional narrowing). Lastly, the BAP mechanism is given by the exchange interaction between electrons and holes. Electronic spins move in an effective field produced by the hole spins, and relaxation takes place when hole spins change in a rate much faster than the electron precession frequency.

For the purpose of QD quantum computing, electrons are individually confined in the QDs or around donor nuclei. This quantization of the electron orbital motion should significantly reduce the cross-section of the spin relaxation channels discussed above. However, if the confined electrons are close to a heterogeneous interface, the associated electric field would increase the strength of spin-orbit coupling for such electrons (for example, in GaAs, whose lattice lacks inversion symmetry). Overall, spin-orbit coupling is relatively weak in the regime for quantum computing and is less important than at higher temperatures and electron densities. Furthermore, without scattering off phonons or impurities, spin-orbit coupling can actually be useful rather than harmful to quantum computing 63, 64. At this limit, other sources of electron spin decoherence, such as electron-nuclear spin coupling, can be more significant, as we will discuss in the next section.

Phonon-assisted spin flip rates due to spin-orbit coupling in a single electron GaAs QD has been calculated [65]. As discussed above, due to wave function localization, the spin orbit relaxation mechanisms for a free electron (EY and $\mathrm{DP}$ ) are strongly suppressed in a $\mathrm{QD}$, giving a long spin flip time: $T_{1} \approx 1 \mathrm{~ms}$ for $B=1 \mathrm{~T}$ and $T=0 \mathrm{~K}$. It was further noticed that spin relaxation is dominated by the EY mechanism, which yields $T_{1} \propto B^{-5}$ for transitions between Zeeman sublevels in a one electron QD.

These calculations are consistent with recent transport measurements of spin relaxation in both vertical and horizontal QDs 66, 67, 68. Pulses of current were injected into a QD coupled to leads in the Coulomb Blockade regime, where the decay rate from excited states can be measured by analyzing the currents generated by the pulses. The results indicate that, for $T=150 \mathrm{mK}$ and $B=0-2 \mathrm{~T}$, spin relaxation times $\left(T_{1}\right)$ are longer than at least a few $\mu$ s in a many-electron QD (less than 50 electrons), and are longer than $50 \mu \mathrm{s}$ in single electron dots. This is encouraging from the perspective of the spin-based solid state QC architectures where spin relaxation times of $\mu$ s or longer are most likely necessary for large scale QC operation. 
It is important to keep in mind that $T_{2}$ is a more directly relevant decoherence time for quantum computing. Therefore experimental determination of electron and nuclear spin $T_{2}$ in semiconductor nanostructures are crucial, but are still to be performed. ESR combined with transport techniques in principle could be used to probe $T_{2}$ in a QD in the Coulomb Blockade regime, just like transport techniques were used to detect ESR in two dimensional electron systems [69]. For example, it was proposed [70] that by applying an AC pump field to a single electron QD subjected to a magnetic field, the stationary current through this QD will exhibit a peak as a function of the pump frequency, whose width will yield a lower bound on $T_{2}$.

\subsection{Spectral Diffusion for Electron Spins}

Spin-orbit coupling does not lead to pure dephasing effect, so that one should expect to have $T_{1}=2 T_{2}$ in materials where spin-orbit coupling in combination with phonon/impurity scattering dominates the spin relaxation, such as high quality GaAs at higher electron density. When the strength of spinorbit coupling or the cross-section of scattering is reduced, other possible spin decoherence channels have to be considered.

In GaAs, all nuclear isotopes $\left({ }^{69} \mathrm{Ga},{ }^{71} \mathrm{Ga}\right.$, and $\left.{ }^{75} \mathrm{As}\right)$ have spin $3 / 2$, and there is finite hyperfine interaction between conduction electrons and all these isotopes [71. The hyperfine interaction is essentially an on-site dipole-dipole interaction between electron and nuclear spins and is $\propto \mathbf{S} \cdot \mathbf{I}$, which includes terms that lead to simultaneous spin flip-flop of both electron and nuclear spins. At a finite magnetic field, the Zeeman splittings of electron and nuclear spins are different by three orders of magnitude, thus energy conservation requires another process to be involved in the transition, therefore reducing the cross-section of such type of processes [72. However, in the hyperfine interaction there is also a term that is proportional to $S_{z} I_{z}$, where the nuclear spins basically produce an effective magnetic field for the electron (assuming one electron trapped in a QD in our situation). If the nuclear spins are all frozen in their respective states, they would simply produce a random but fixed field, which would result in the so-called inhomogeneous broadening for the electron and the effect can be accounted for by calibration and spin echo techniques 59, 60. If the nuclear spins are dynamically coupled (through dipolar coupling, for example), though, the trapped electron would be in a magnetic field that fluctuates both spatially and temporally. This fluctuating field makes the electron Zeeman splitting a random variable that undergoes the so-called spectral diffusion, which results in pure dephasing for the electron spin 73. The calculated results for GaAs QDs are in the order of tens of $\mu \mathrm{s}$.

Theoretically, it can be envisioned that if all the nuclear spins are polarized, the corresponding electron spin spectral diffusion and dephasing can be suppressed [54]. However, 100\% polarized nuclear spins would create a significant effective magnetic field, which can have its own negative side effect. 
Furthermore, the creation of this high degree of polarization is also nontrivial. In other words, ingenious approaches need to be devised to deal with the nuclear spins in a GaAs QD, and we may not have seen the optimal approach just yet.

Spectral diffusion can occur in donor electrons in $\mathrm{Si}$ as well, when the host Si material is not purified: Naturally occurring Si contains more than $95 \%$ of ${ }^{28} \mathrm{Si}$ and ${ }^{30} \mathrm{Si}$, which have no nuclear spin, and nearly $5 \%{ }^{29} \mathrm{Si}$, which has nuclear spin $1 / 2$. For a confined donor electron (at a phosphorus site, for example), which has a Bohr radius about $30 \mathrm{~nm}$, there can be a lot of nuclear spin- $1 / 2 \mathrm{~s}$ (more than $10^{4}$ of them within a sphere of radius of $30 \mathrm{~nm}$ ) with finite hyperfine coupling to the electron, and thus can produce spectral diffusion in this electron as discussed above [73. Fortunately, in Si there is a way to reduce the effect from the nuclear spins of ${ }^{29} \mathrm{Si}$ : isotopic purification. If a complete purification can be achieved, the nuclear spin induced electron spin spectral diffusion can be completely suppressed [73].

\section{Spin Manipulations and Exchange}

\subsection{Spin Hamiltonian in a GaAs Double Quantum Dot: Coulomb Interaction and Pauli Principle}

One of the key issues in the spin-based QD QC involving exchange coupling in a coupled QD is to accurately describe the electron interaction in terms of a spin Hamiltonian such as a Heisenberg exchange Hamiltonian with appropriate correction terms. Ideally, for small QDs at low temperatures, the electron orbital degrees of freedom are frozen, so that the only states two electrons can possibly occupy in a double dot are the ground spin singlet and triplet states, whose splitting is the exchange splitting $J$. However, it is important to clarify whether the ground state manifold is well separated from the excited states, and whether the exchange splitting $J$ in the ground state manifold is sufficiently large to support a practical QC.

The Hamiltonian for two electrons in an electrostatic confinement produced by surface gates and growth direction barriers can be written as

$$
H=\sum_{i=1,2}\left[\frac{1}{2 m^{*}}\left(\mathbf{p}+\frac{e}{c} \mathbf{A}\left(\mathbf{r}_{i}\right)\right)^{2}+V\left(\mathbf{r}_{i}\right)\right]+\frac{e^{2}}{\epsilon r_{12}}+\sum_{i=1,2} g^{*} \mu_{B} \mathbf{B}\left(\mathbf{r}_{i}\right) \cdot \mathbf{S}_{i} .
$$

This is an effective mass Hamiltonian where the underlying Bloch function at $\Gamma$ point (the bottom of the GaAs conduction band) is already factored out. Notice that none of the spin-dependent terms except Zeeman coupling are included in this Hamiltonian. In essence, electrostatic interaction is much stronger than direct magnetic interactions such as magnetic dipole interaction. Neglected interactions include electron spin-orbit interaction (which will be discussed later on in this subsection), electron-electron magnetic dipole 
interaction, electron-nuclear spin contact hyperfine interaction (discussed in the previous section on electron spin spectral diffusion), electron-nuclear spin dipole interaction, and other higher order interactions. In fact, in arriving at the effective mass Hamiltonian (6), small spin-mixing terms inversely proportional to the conduction band gap are also neglected [74].

Here we have separated the two-electron-interaction related terms of the Hamiltonian from those that involves interactions between either or both of the electrons and the surrounding environments that include the crystal lattice (in terms of phonons), the nuclear spins of the ions, and other electrons present in the system. The effects of these interactions are categorized as decoherence, in the sense that as soon as electron spin coherence passes into these channels, the chance of a revival of the coherence is vanishingly small.

The theoretical calculation of electron spin exchange in a double dot was first done using the Heitler-London approach, in which the electron orbital states are limited to the ground states in the two single QDs that form the double dot [54, and double occupied single dot states are excluded. The effects of the lowest two double-occupied states (where both electrons are in the same dot) are included in the so-called Hund-Mulliken calculation [54. Both calculations indicated that the exchange constant can be sizable in a GaAs QD system.

More accurate calculations of the exchange coupling and the overall spectrum of the two QD-confined electrons can be performed with larger basis of single dot states. For example, we performed a molecular orbital calculation to further clarify the properties of the exchange splitting in a GaAs horizontal double QD by including the excited P orbital states of the two QDs [55]. The inclusion of the anisotropic $\mathrm{P}$ orbitals provides more flexibility to electron wavefunction deformation and bonding, thus leads to more faithful description of the exchange splitting of the two-electron ground states. After all, molecular bonding is strongly affected by electron distribution in space, while the system we considered is essentially an effective two-dimensional hydrogen molecule. The system we considered is formed from two-dimensional electron gas by surface gate depletion (the so-called horizontal or lateral QDs). The growth direction confinement (due to AlGaAs alloys) is so strong that the excitation energy scale along that direction is much higher than the horizontal direction and excitation along that direction is neglected (thus the name horizontal or lateral QD). One important advantage of the horizontally coupled QDs is that the inter-dot coupling can be easily tuned with surface gate potential adjustments. Our numerical results showed that the inclusion of the $\mathrm{P}$ orbitals indeed affect the exchange coupling quite significantly, generally causing an increase about $20 \%$ compared to the Hund-Mulliken calculation [55] (see Figs. 3] and 4). The size of the QDs we considered is quite small (in the order of 40 to $50 \mathrm{~nm}$ in diameter), somewhat smaller than the state of the art experimental value of about $100 \mathrm{~nm}$. The increase in size invariably 
leads to smaller on-site Coulomb repulsion, smaller single particle excitation energy, and generally smaller exchange coupling.

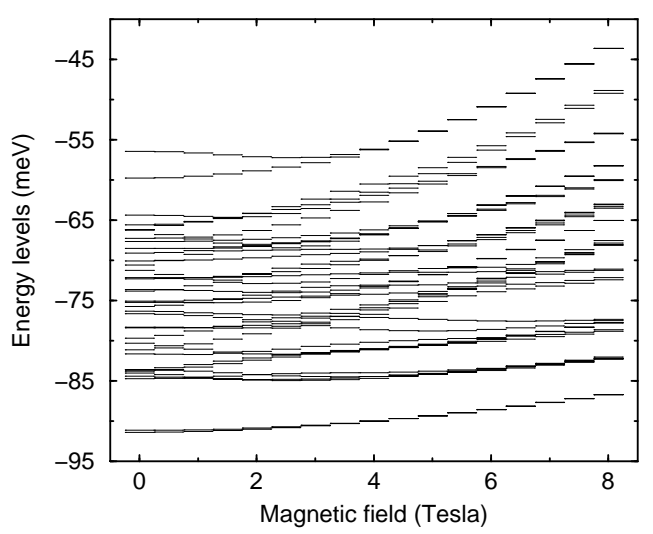

Fig. 3. Two-electron energy spectrum of a GaAs horizontal double QD [55] as a function of applied perpendicular magnetic field. The ground singlet-triplet manifold is well separated from the excited states. Here the inter-dot distance is $30 \mathrm{~nm}$, and the ground electron wavefunction radius is about $10 \mathrm{~nm}$.

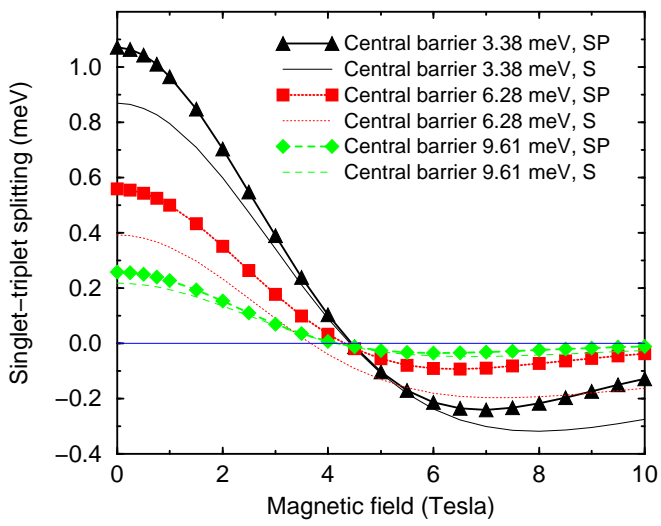

Fig. 4. Exchange splitting between the ground singlet and unpolarized triplet states of a GaAs horizontal double QD [55]. 
The spectroscopic results for double QD [54, 55] show that exchange coupling should be sufficiently strong to implement quantum computing operations, where a basic controlled-NOT operation can be built from single qubit spin rotations and two so-called square-root-of-swap gates [10:

$$
U_{C N O T}=e^{i \frac{\pi}{4} \sigma_{2 y}} e^{i \frac{\pi}{4} \sigma_{1 z}} e^{-i \frac{\pi}{4} \sigma_{2 z}} U_{s w}^{\frac{1}{2}} e^{i \frac{\pi}{2} \sigma_{1 z}} U_{s w}^{\frac{1}{2}} e^{-i \frac{\pi}{4} \sigma_{2 y}},
$$

where $\sigma$ are Pauli matrices, and $U_{s w}=\exp \left(i \pi \sigma_{1} \cdot \sigma_{2}\right)$. For example, an exchange splitting of $0.1 \mathrm{meV}$ corresponds to a swap gate with duration as short as 100 ps. Compared to the electron spin decoherence time that might be as long as milliseconds, such gating time is sufficiently short for quantum error correction codes to work properly.

By including only the lowest spin singlet and triplet states, we have implicitly assumed that the higher energy two-electron states can be neglected. Such an assumption is based on the electron states being manipulated adiabatically. On the other hand, the gate operations are limited in duration by the electron spin decoherence time. It is thus imperative to determine what the adiabatic requirement is in the present architecture. Such calculation was carried out within the Hund-Mulliken model by assuming a particular temporal shape of the exchange splitting $J$ in a double QD [75]. We performed such a calculation with calculated two-electron energy spectra and wavefunctions [76]. Instead of assuming a particular temporal profile for the exchange coupling $J$, we assumed a temporal variation in the inter-dot barrier height, which is a quantity that can be directly tuned by external voltages applied to the surface gates. Our results show that errors due to non-adiabaticity decreases rapidly as the gate operations become longer, and the requirement for adiabatic operation is not overbearing on the spin-based GaAs QD QC [76.

The calculations on the energy spectra and adiabatic manipulation thus justify the use of Heisenberg spin exchange Hamiltonian to describe the low energy dynamics in a two-electron double QD (whose effective mass Hamiltonian is given by Eq. 6) and the related two-qubit operations in a spin-based QD QC. In these calculations spin-orbit coupling has been neglected because of their small magnitude for the conduction electrons in bulk GaAs (near the bottom of the conduction band the electron wavefunction is mostly formed from the atomic S orbitals). However, the horizontal QDs are made from two-dimensional electron gas confined in heterostructures or quantum wells, where the sharp surfaces and asymmetry lead to strong Rashba type spinorbit coupling (even for symmetric quantum wells, this spin-orbit coupling does not vanish because of the lack of inversion symmetry in GaAs), which in turn leads to finite anisotropic exchange in the spin interaction [77] in the form of $\mathbf{h} \cdot\left(\mathbf{S}_{1} \times \mathbf{S}_{2}\right)$ plus higher order corrections. Here $\mathbf{h}$ is a vector determined by spin-orbit interaction. The inclusion of these terms does not take away the capability of QDs to perform quantum logic operations (indeed, there has been a proposal to use the anisotropic exchange coupling 
to perform quantum logic operations 63, 64]). Nevertheless, they do add more complexity to the aesthetically simple and beautiful isotropic Heisenberg exchange coupling. Fortunately, it has been proved that by carefully choosing the temporal profile of the inter-dot coupling (basically maintaining time reversal symmetry), it is possible to largely eliminate the effects of the anisotropic exchange due to the spin-orbit interaction [78].

Even when the two-electron interaction in a double dot can be characterized by the Heisenberg spin exchange Hamiltonian, inhomogeneity in the single spin environment can still cause problems in the two-qubit quantum logic operations. For example, we showed that inhomogeneous Zeeman coupling leads to incomplete swap operations [79. This means that swap cannot be accomplished by a single pulse of exchange gate anymore. Instead, several pulses (at least 3) have to be used for large inhomogeneity [80, while smaller inhomogeneities such as those due to trapped charges nearby can be and have to be corrected [79]. Interestingly, inhomogeneous Zeeman coupling can also be utilized for the purpose of qubit encoding [81, which leads to all-exchange logical operations (eliminating the need for local magnetic field and/or g-factor engineering) [82 that originate from the concept of decoherence free subspace [83].

To relax the requirement of spin-based QD quantum computation, multielectron QDs have also been studied as candidates for qubits [84. For instance, we performed a configuration interaction calculation for a double dot with six electrons (three per dot) to explore whether the low-energy dynamics is still entirely dominated by spin dynamics. Our results showed that the ground state complex is well separated from the higher energy excited states (Fig. 5) at relatively high magnetic fields, and the splitting between the lowest-energy singlet and triplet states can be sizable as well (Fig. [6). However, our results also showed that orbital level degeneracy can lead to the participation of multiple states in the low energy dynamics at zero or low magnetic fields, therefore causing serious complexity and difficulty in spin exchange. To solve this problem, external means such magnetic field or quantum dot deformation has to be applied to lift the orbital degeneracy so that the electron cloud in each QD can again be described by an effective spin-1/2 entity. Another theoretical study has also clearly demonstrated a variety of difficulties in the control of gate operations when one attempts to use multi-electron QDs as qubits [85].

In using a tunable exchange interaction for quantum gates, one needs to control the magnitude and duration of the interaction precisely. Since exchange interaction depends sensitively on the wavefunction overlap, its precise control is of critical importance. One suggestion on how a good control can be achieved is to control the way the wavefunction overlap is tuned by altering the geometry of the QD designs and is called a pseudo-digital approach [86]. As the experimental study of QD QC pushes toward two and more qubits and qubit manipulations, such considerations are directly relevant to the de- 


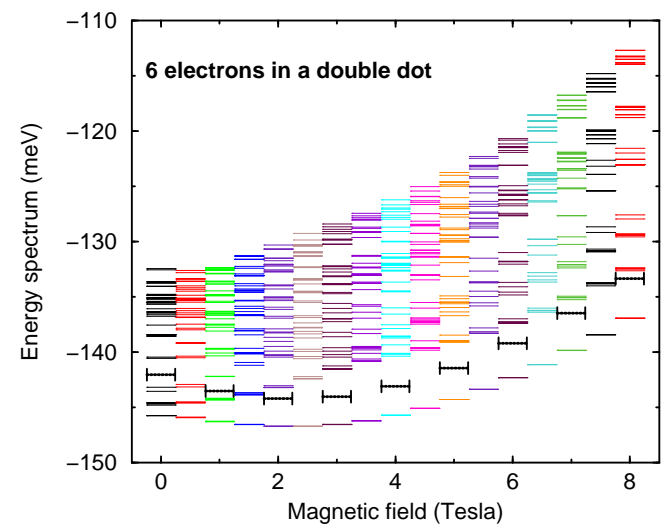

Fig. 5. Six-electron energy spectrum of a GaAs horizontal double QD 84 .

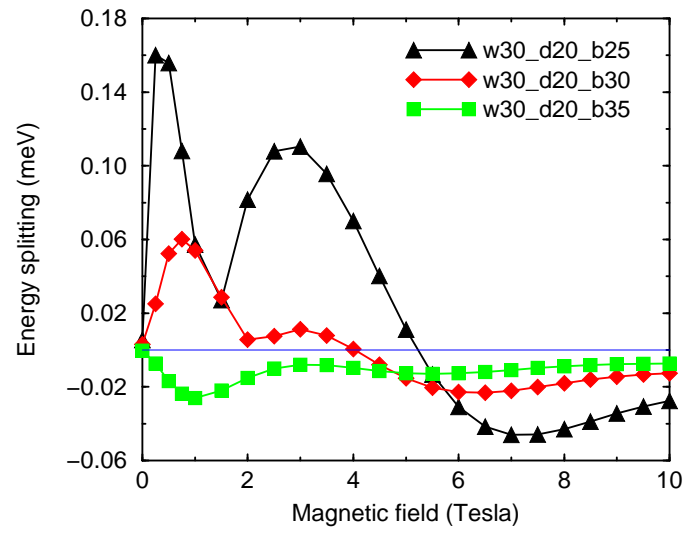

Fig. 6. Energy splitting of the two lowest energy states of a six-electron GaAs horizontal double dot 84 .

signs of next generation architectures and ultimately the scale-up of any of the QD-based QC schemes.

\subsection{Implications of Si Conduction Band Structure to Electron Exchange}

As we mentioned before, $\mathrm{Si}$ possesses a variety of nice material properties (small spin-orbit coupling, spinless isotopes, etc.) for the purpose of quantum computing, so that it is clearly one of the favored host materials for a solidstate QC. However, Si does have one complexity that GaAs, the other popular 
semiconductor material, does not have: The Silicon conduction band has six minima close to the X points of the silicon First Brillouin Zone 87, so that donor electron wavefunctions have to be expanded on the basis of the six Bloch functions at these points. It was pointed out in the context of donor magnetic phase transition that the presence of degenerate conduction valleys leads to valley interferences and a shift to smaller values for average electron exchange coupling 88 . The potential problem such interference effects may cause to a donor based Si QC was also mentioned in the original proposal 12.

To quantitatively address this concern over donor exchange coupling in $\mathrm{Si}$, which is a crucial link for two-qubit operations in the Si QC architecture, we have performed a series of Heitler-London type calculations of the donor electron exchange coupling. Such a calculation is based upon the single donor wavefunctions, which can be expressed as [87, 88, 89, 90.

$$
\psi(\mathbf{r})=\sum_{\mu=1}^{6} \alpha_{\mu} F_{\mu}(\mathbf{r}) \phi_{\mu}(\mathbf{r})=\sum_{\mu=1}^{\mathbf{6}} \alpha_{\mu} \mathbf{F}_{\mu}(\mathbf{r}) \mathbf{u}_{\mu}(\mathbf{r}) \mathbf{e}^{\mathbf{i} \mathbf{k}_{\mu} \cdot \mathbf{r}},
$$

where $F_{\mu}$ are the so-called envelope functions while $\phi_{\mu}(\mathbf{r})$ are the Bloch functions at the bottoms of the Si conduction band [89]. $\left|\mathbf{k}_{\mu}\right| \approx 0.85 \cdot 2 \pi / a$ is the location of the conduction band minima and is very close to the $\mathrm{X}$ points. The presence of these plane wave phase factors leads to a significantly more complicated expression (compared to, for example, GaAs) for the electron exchange splitting [90]:

$$
J(\mathbf{R})=\sum_{\mu, \nu}\left|\alpha_{\mu}\right|^{2}\left|\alpha_{\nu}\right|^{2} \mathcal{J}_{\mu \nu}(\mathbf{R}) \cos \left(\mathbf{k}_{\mu}-\mathbf{k}_{\nu}\right) \cdot \mathbf{R} .
$$

Here $\mathcal{J}$ represents integrals over the envelope functions and is thus a slowly varying function of donor positions. The key fact here is that the $\mathbf{R}$ dependence of $J(\mathbf{R})$ is strongly oscillatory because of the sinusoidal factors $\cos \left(\mathbf{k}_{\mu}-\mathbf{k}_{\nu}\right) \cdot \mathbf{R}$.

Our numerical results showed that the inter-valley interference indeed leads to strong atomic scale oscillations in the inter-donor electron exchange (see Figs. 7 and 8), which potentially presents a significant difficulty in the control of two-qubit operations [89. Uniaxial strain can be used to break the Si lattice symmetry and partially lift the degeneracy between the valleys, so that as few as two valleys make up the bottom of the conduction band. Then the sum over $\mu$ in Eq. (9) is much simplified, but the sinusoidal factor will still remain, so that care still has to be taken in controlling the donor exchange (Figs. 9 and 10) 90. These results have been corroborated by another calculation that also considered higher order corrections in Coulomb interaction energy 91. More recently we have attempted to relax the HeitlerLondon approximation to minimize the two-electron energy 92. However, the results showed that in the cases of donors, which have very low single particle 
potential energy near the donor nuclei, the two-electron contribution to total energy is completely dominated by the single particle contributions, thus the Heitler-London results based on single donor states are quite robust 92 .

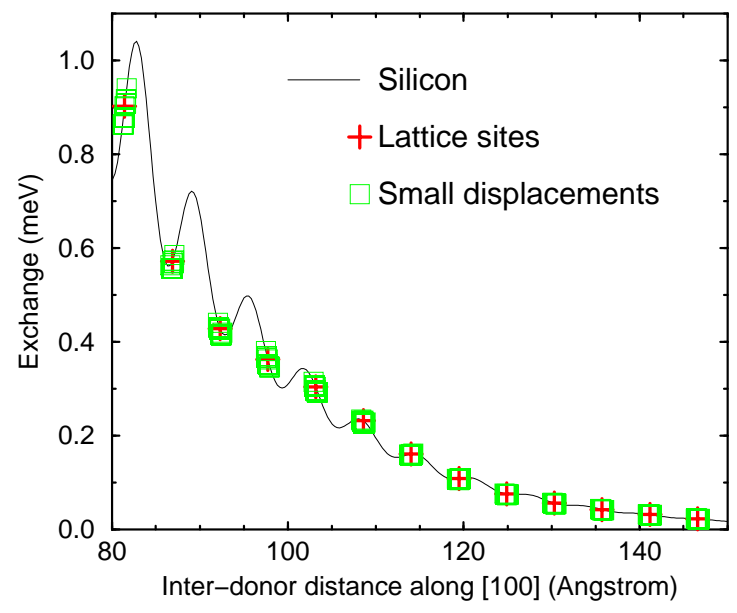

Fig. 7. Donor electron exchange splitting in relaxed bulk Si. The two ${ }^{31} \mathrm{P}$ donors are aligned along the [100] direction [89].

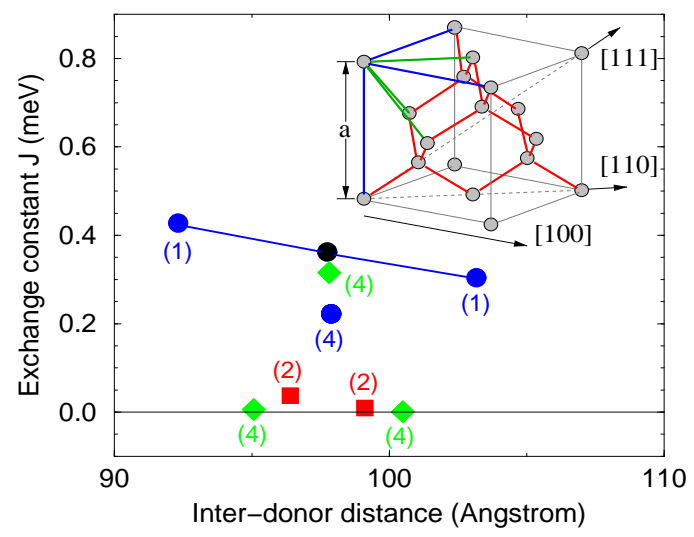

Fig. 8. Donor electron exchange splitting in bulk Si. The two ${ }^{31} \mathrm{P}$ donors are almost aligned along the [100] direction, separated by about 18 lattice constants, and with one of the donors displaced into its nearest neighbor lattice sites [89]. 


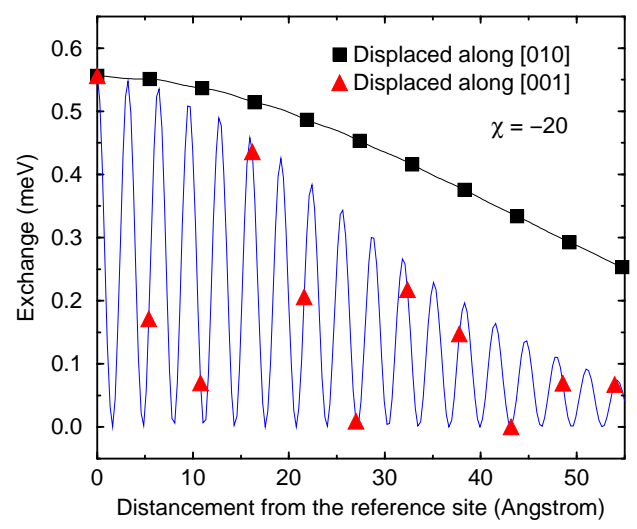

Fig. 9. Donor electron exchange splitting in Si uniaxially strained along [001] direction $(\chi=-20)$. The two donors are approximately aligned along the [100] direction, with one of them displaced along the [010] direction [90].

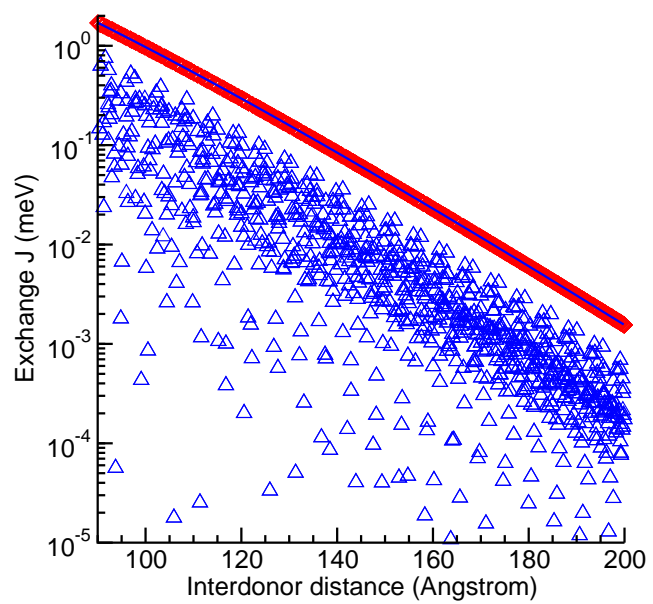

Fig. 10. Donor electron exchange splitting in both relaxed bulk Si and Si strained along the [001] direction. The two donors are both in the (001), or $x y$, plane. We consider a situation where one of the donors is located in any of the possible lattice positions between two concentric circles of radii $90 \AA$ and $180 \AA$ with the other donor positioned at the center of the circles. The data points correspond to the exchange calculated at all relative positions considered. The solid line is $J(\mathbf{R})$ for $\mathbf{R}$ along the [100] direction for $\chi=-20$ [90]. 
As we mentioned before, there have also been proposals using electron spins in Si or SiGe host materials for quantum computing. The problem with oscillatory exchange also plagues the donor-electron-based scheme. In the case of QDs in $\mathrm{Si}$, the problem becomes more subtle. In such a system there does not exist a strong scattering center such as a donor, so that the different valleys in Si do not couple significantly, so that the validity of Eq. (8), with its strongly pinned phase for each valley, becomes questionable. The valley degeneracy is lifted by the quantum well for the QDs, but the energy splitting is quite small [93. More theoretical and experimental studies are needed to further clarify this situation.

One way to avoid the potential problems associated with the control of inter-donor exchange coupling is to move a donor electron around between ionized nulei 94, 95. Here nuclear spins are still the qubits, while a single electron is used as a shuttle to physically move among nuclei to enable effective two-qubit coupling and operations. Since on-site hyperfine coupling has been shown to be quite robust against Si bandstructure complexities 96, 97, this approach thus maintains the long coherence time advantage of a donor nuclear spin in $\mathrm{Si}$ while completely removes the requirement of inter-donor exchange coupling. However, a potential disadvantge of such a charge-coupled device may be associated with the charge movement and the corresponding spin decoherence. Further analysis is still needed to clarify the physical picture here.

\subsection{Single Spin Detection Schemes}

Single spin detection is crucial for any spin-based QC architecture to work properly, both in terms of quantum error correction, and in terms of reading out the final results of a calculation. However, since magnetic force is weak (compared to electrical force), and a single Bohr magneton is a very small magnetic moment, direct measurement within a short duration (such as using the most sensitive magnetometer available at present, a SQUID magnetometer) is almost impossible. However, several techniques are being actively studied and have produced some promising progresses.

One approach to single spin detection is to first convert the electron spin states into electron charge states, for example through spin blockade effects 98, 99, 100, 101. It is well established now that single electron transistors (SET) and quantum point contacts (QPC) are extremely sensitive charge detectors 102. Thus, if one can establish a correlation between electron charge location and spin states, a means to determine spin states can be established by observing the current or conductance of the SET/QPC charge detector.

One shortcoming of the conventional DC-biased SETs and QPCs is that they are relatively slow detectors, with bandwidth in the order of $10 \mathrm{kHz}$. However, an alternative approach to DC-SET has been proposed [103. Instead of measuring current directly, here pulsed radio-frequency field is sent into a circuit containing an SET and the circuit response is measured. The relevant 
physical quantity in such a measurement is conductance, which can be determined without collecting a large number of electrons. The bandwidth of these so-called Radio Frequency SETs (RF-SET) has been shown to reach above $10 \mathrm{MHz}$, so that they are very promising candidates for quantum detectors. Now, if spin states can be efficiently converted to charge locations, single spin detection also becomes possible. One of the early examples of such conversion was suggested in connection with the donor in Si architecture, where the onsite exchange splitting between a singlet and a triplet state on a double valent donor, together with an SET charge detector, is used for spin detection [57]. A similar scheme can also be constructed for artificial QD-based structures.

Quantum measurement of hyperfine split nuclear spin levels in trapped ion systems has been achieved using the so-called quantum jump technique, in which an ancilla electron orbital level and light absorption/emission between this level and the qubit levels are used to read out the quantum state of the qubit with basically $100 \%$ efficiency. Such ancilla levels certainly also exist for semiconductor QDs, so that similar quantum jump processes have been suggested for single electron spin detection [47. Concrete examples of such a scheme have been described and simulated for an electron spin in a QD 104 105.

In the original proposal for $\mathrm{QD} \mathrm{QC}$ in GaAs, the so-called spin valve effect is suggested for spin measurement [10], where electron tunneling into another QD depends on its spin orientation, in analogy to giant magnetic resistance [106. An alternative is to prepare a supercooled paramagnetic dot, so that when an electron tunnel into this QD, a large ferromagnetic domain could quickly nucleate along the incoming spin direction. This more macroscopic magnetization can then be detected in a more traditional way [10].

Another approach toward spin measurement is a direct magnetic force detection using the so-called magnetic resonance force microscopy (MRFM) 107. In MRFM a small magnet at the tip of a cantilever creates a strongly inhomogeneous magnetic field near the surface of a sample containing paramagnetic electron spins. When a RF field with a certain frequency is applied, only those spins that are Zeeman split by the right amount can get into resonance with the external RF field, so that these spins can apply controlled forces on the cantilever. By measuring the motion of the cantilever, one can infer information of those electron spins that are on resonance (thus the name MRFM).

Single spin detection is not needed for the purpose of characterizing a single or double QD/donor system. For example, we proposed a scheme to use resonant micro-Raman scattering to measure inter-donor exchange coupling 108. The polarization- and temperature-dependence of Raman scattering provides abundant information of a two-donor system, while resonant photons enhances the cross-section of such scattering process so that single pairs of donors can be observable. Similarly, transport and/or optical techniques can 
be used to measure the ground exchange splitting in a double QD as well [109.

\subsection{Approaches to generate and detect electron spin entanglement in quantum dots}

Two basic ingredients of spin-based QD QC are single spin manipulation and particularly single spin detection. These are very hard tasks and are attracting plenty of attention from experimentalists and theorists alike. In the meantime, traditional ensemble-averaged experiments can also be used to demonstrate quantum coherent properties of electron spins. For example, electron spin entanglement has never been experimentally demonstrated in condensed matter systems in terms of measured correlations, because of the usual presence of strong interaction and the associated difficulty in isolating the target electrons. However, with the help of QDs and transport through them, it is possible to generate and detect electron spin entanglement. From the perspective of $\mathrm{QD} \mathrm{QC}$, a reliable source of spin entangled electrons is very important to tasks such as error correction.

Many approaches for creating/detecting entanglement in solid state systems have been theoretically proposed in the literature. For example, Cooper pairs in a superconductor are (usually) in a spin singlet (and entangled) state, thus it is quite natural to consider extracting them with control to make up a source of pairs of spin-entangled electrons [110, 111, 112, 113, 114, 115. The key here is to separate the two electrons into different drain electrodes using, for example, Coulomb blockade in quantum dots or wires. There are many other physical systems within solid state that have electron spin entangled states as part of their eigenstate spectrum and have been suggested as sources of entangled electrons. Examples include quantum dot and quantum wire electron singlet states in semiconductors [116, 117, 118, 119, 120. Here we briefly discuss our idea of using a double dot to create spin entangled electron pairs [119.

As discussed in the previous sections, at zero or low magnetic field, the ground state of a two-electron double dot is a spin singlet state, where the spins of the two electrons are entangled $((|\uparrow \downarrow\rangle-|\downarrow \uparrow\rangle) / \sqrt{2})$. However, sequential tunneling through a double dot is generally dominated by the lowest order processes, so that the current through a double dot is generally made up of mostly single electrons tunneling out of the entangled state. To extract pairs of spin-entangled electrons, a relatively straightforward approach is to introduce time-dependent (specifically, periodic) tunnel barriers, so that during part of one cycle the electrons form molecular states (such as the spin singlet state) in the double dot, while during the rest of the cycle the electrons are emptied out into the drain electrodes. Such time-dependent manipulation of tunnel barriers is quite well-understood for single quantum dots, and in principle feasible for a double dot. We estimated various parameters for such a 
double dot turnstile and found that they are quite reasonable and fall within the capability range of currently available technology.

A possible method to observe solid state electronic entanglement within ensemble-averaged approach is to use two-electron interference in transport. One example is to use a beam splitter [121, 119, into which pairs of entangled electrons are injected. Although this detection scheme is not a true Belltype measurement, it is nonetheless an important first step as it deals with correlations between electrons that have been extracted from their entangler and separated.

\section{Current Experimental Status}

\subsection{Single electron trapping in horizontal QDs}

Since the invention of gated semiconductor QDs, there has been a continuous trend to fabricate structures that can hold smaller and smaller number of electrons. Single electron QD through electrical transport was achieved in vertically contacted InGaAs QDs several years ago 122. However, since confinement in a gated dot is given by an electrostatic field produced by metallic gates that are 50 to $100 \mathrm{~nm}$ away, and the feature width of a metallic gate is generally above $20 \mathrm{~nm}$, horizontal QDs made from depleted two-dimensional electron gas are generally large in size but energetically shallow. In these QDs it is difficult to simultaneously increase depletion (to reduce number of trapped electrons) and maintain a finite coupling to the source and drain leads (so that transport characterization can be done). A solution to this problem was found several years ago, when a new design with source and drain coupling controlled by a single gate was studied [123]. The design has since been used to create single electron QDs and two-electron double QDs successfully [124, 125]. Furthermore, recent experiments have shown that electrons trapped in such a QD have significant (and consistent with expectation) Zeeman splitting, and that spin-flip time is extremely long in these confined states 68 .

\subsection{Single spin detection}

Although many theoretical proposals have been published on how to achieve single spin detection, experimental demonstration remains a challenging and hotly pursued goal, particularly for solid state spin-based QC architectures, though impressive progress have been made along a variety of lines of research.

Recently, QPCs have been used to successfully measure single QD properties such as QD charging and excited state spectroscopy [125] 126]. The simpler structure of a QPC makes it an enticing alternative to an SET detector, and may be easier to integrate into larger structures. 
In analogy to the quantum jump technique in trapped ion QC schemes [6], optical transitions have been used to observe single spin states of nitrogenvacancy defect centers in diamond [127]. These observations clearly demonstrated that although solid state systems general have much more complexity compared to atomic systems, many of the techniques and concepts can be transferred with truly fruitful consequences.

It has recently been reported that as few as two electron spins can be reliably detected with a particular realization of the MRFM [128]. Spin diffusion suppression, which is intimately related to the interaction between the cantilever and the spin being observed, has also been characterized in the inhomogeneous magnetic field produced by the small magnet at the end of the cantilever of an MRFM [129].

\subsection{Electron-nuclear spin interaction in QDs}

As we mentioned previously, electron-nuclear spin hyperfine coupling can lead to electron spin spectral diffusion and pure dephasing. Thus it is an important environmental issue for electron spins, especially for QD host materials that have a lot of nuclear spins, such as GaAs and InGaAs (the preferred materials for gated horizontal and vertical QDs, respectively).

There have been quite extensive experimental studies of electron-nuclear spin coupling in bulk semiconductors and more recently semiconductor heterostructures 48, 130, 131, 132. Dynamical nuclear spin polarization has recently been demonstrated in a spin blocked semiconductor double QD made of $\mathrm{Ga}_{0.95} \mathrm{In}_{0.05} \mathrm{As}$ 109, 132, 100. Coulomb interaction and Pauli principle means that in a double QD two-electron singlet and triplet states are split by the exchange interaction, so that proper voltage offset and bias between the double dot leads to significant suppression in the tunnel current due to occupied triplet state (thus the so-called spin blockade regime, which has been suggested for single spin detection, a crucial component of quantum information processing) [100. One way to lift this blockade is to apply an appropriate magnetic field, so that singlet-triplet spin-flip transition can be facilitated by the electron-nuclear spin hyperfine coupling as one of the polarized triplet state is energetically degenerate with the singlet state [132]. This selective transition can then dynamically polarize the nuclear spins in the double QD. Indeed, strong experimental evidences have been observed that nuclear spins are indeed being polarized 132, though many physical issues still have to be sorted out before a more thorough understanding of the coupled electron-nuclear spin system can be achieved.

\subsection{Fabrication of donor arrays in $\mathrm{Si}$}

Currently there are active experimental research efforts in attempting to fabricate well-controlled ${ }^{31} \mathrm{P}$ donor arrays in a $\mathrm{Si}$ crystal, and plenty of experimental progress have been made [133. Two experimental approaches are 
adopted, attacking the problem from opposite directions 134 133. One uses ion implantation by bombarding ${ }^{31} \mathrm{P}$ ions into crystalline silicon, thus it is also called a top-down approach. The other uses MBE to grow the system layer by layer and STM to identify donor locations, and is called the bottom-up approach.

In the top-down approach, annealing is needed after the bombardment to make the ${ }^{31} \mathrm{P}$ donors substitutional so that they become shallow donors. If they remain interstitial, they would behave as deep centers [135, which have different electronic structures and thus are not useful for the purpose of quantum computing within the Kane proposal. Single electron transistors are used to monitor the presence of donors since they are very sensitive to net charges 133. At present the precise positioning of the donors and annealing of the Si host lattice are being actively studied [136, 137.

In the bottom-up approach, a clean Si surface is first hydrogenated. An STM is then used to pick off hydrogen atoms at desired locations, after which the surface is doused with $\mathrm{PH}_{3}$ gas so that phosphorus atoms would tend to attach to the surface at the vacancies. This way an ordered array of donors can be fabricated with a high degree of regularity. Using this approach, it has been shown that precise positioning of phosphorus donors into a linear array can be achieved on a $\mathrm{Si}$ [001] surface [138. More recently, the incorporation of the donors into the bulk of Si has also been demonstrated [139]. Athough much more experimental efforts have to be invested, and most probably more sophisticated technologies in donor positioning and manipulation have to be invented for this QC architecture to be fabricated with precision, recent experimental progresses are nonetheless quite impressive and promising.

\section{Summary}

We have presented a brief review of the theoretical and experimental progresses related to spin-based QD QC architectures. We introduced the most prominent proposals of QD QC, outlined the basics on how these proposals might work, explored potential problems with the different material systems, and finally discussed where the present experimental studies stand. We thank US ARO, ARDA, and LPS for continued financial support to our research. The results presented here are the products of collaborations with S. Das Sarma, B. Koiller, D. Drew, R. de Sousa, R.B. Capaz, and fruitful discussions with J. Fabian, I. Zǔtić, A. Kaminski, M. Friesen, R.A. Webb, Y.Z. Chen, B.E. Kane, D. Romero, S. Tarucha, K. Ono, R. Hanson, and R. Budakian.

\section{References}

1. D. DiVincenzo, Science 270, 255 (1995); A. Ekert and R Jozsa, Rev. Mod. Phys. 68, 733 (1996); A. Steane, Rep. Prog. Phys. 61, 117 (1998). 
2. M. Nielsen and I.L. Chuang: Quantum Computation and QUantum Information (Cambridge, Cambridge 2000).

3. P.W. Shor, in Proceedings of the 35th Annual Symposium on the Foundations of Computer Science, ed. by S. Goldwasser (IEEE Computer Society, Los Alamitos, 1994), p. 124.

4. P.W. Shor, Phys. Rev A 52, 2493 (1995); A.M. Steane, Phys. Rev. Lett. 77, 793 (1996).

5. J.I. Cirac and P. Zoller, Phys. Rev. Lett. 74, 4091 (1995).

6. C. Monroe, D.M. Meekhof, B.E. King, W.M. Itano, and D.J. Wineland, Phys. Rev. Lett. 75, 4714 (1995).

7. T. Sleator and H. Weinfurter, Phys. Rev. Lett. 74, 4087 (1995).

8. Q.A. Turchette, C.J. Hood, W. Lange, H. Marbuchi, and H.J. Kimble, Phys. Rev. Lett. 75, 4710 (1995).

9. D.G. Cory, A.F. Fahmy, and T.F. Havel, Proc. Natl. Acad. Sci. U.S.A. 94, 1634 (1997); N.A. Gershenfeld and I.L. Chuang, Science 275, 350 (1997).

10. D. Loss and D.P. DiVincenzo, Phys. Rev. A 57, 120 (1998).

11. A. Imamoglu et al: Phys. Rev. Lett. 83, 4204 (1999).

12. B.E. Kane: Nature 393, 133 (1998); Fortschr. Phys. 48, 1023 (2000).

13. V. Privman, I.D. Vagner, and G. Kventsel, Phys. Lett. A 239, 141 (1998).

14. R. Vrijen et al: Phys. Rev. A 62, 012306 (2000).

15. A. Shnirman, G. Schön, and Z. Hermon: Phys. Rev. Lett. 79, 2371 (1997); D.V. Averin: Solid State Comm. 105, 659 (1998).

16. D. Leibfried et al: Nature 422, 412 (2003).

17. L.M.K. Vandersypen et al: Nature (London) 414, 883 (2001).

18. A. Barenco, D. Deutsch, and A. Ekert: Phys. Rev. Lett. 74, 4083 (1995).

19. D.A.B. Miller, D.S. Chemla, and S. Schmitt-Rink: Phys. Rev. B 33, 6976 (1986).

20. J.A. Brum and P. Hawrylak: Superlattices and Microstructures 22, 431 (1997).

21. G.D. Sanders, K.W. Kim, and W.C. Holton: Phy. Rev. B 61, 7526 (2000).

22. T. Tanamoto: Physica B 272, 45 (1999).

23. L. Fedichkin, M. Yanchenko, and K.A. Valiev: Nanotechnology 11, 387 (2000).

24. M.D. Lukin et al: Phys. Rev. Lett. 87, 037901 (2001).

25. M.S. Sherwin, A. Imamoglu, and T. Montroy: Phys. Rev. A 60, 3508 (1999).

26. V. Bouchiat et al: Phys. Scripta T 76, 165 (1998).

27. Y. Nakamura, Yu.A. Pashkin, and J.S. Tsai: Nature 398, 786 (1999).

28. D. Vion et al: Science 296, 886 (2002).

29. T. Yamamoto et al: Nature 425, 941 (2003).

30. T. Hayashi et al: Phys. Rev. Lett. 91, 226804 (2003).

31. S.R.E. Yang, J. Schliemann, and A.H. MacDonald: Phys. Rev. B 66, 153302 (2002).

32. V. Scarola, K. Park, and S. Das Sarma: Phys. Rev. Lett. 91, 167903 (2003).

33. P. Zanardi and F. Rossi: Phys. Rev. Lett. 81, 4752 (1998).

34. E. Biolatti et al: Phys. Rev. Lett. 85, 5647 (2000).

35. P.C. Chen, C. Piermarocchi, and L.J. Sham: Phys. Rev. Lett. 87, 067401 (2001).

36. F. Troiani, U. Hohenester, and E. Molinari: Phys. Stat. Sol. B 224, 849 (2001).

37. K.B. Brown, D.A. Lidar, and K.B. Whaley, Phys. Rev. A 65, 012307 (2002).

38. A.P. Heberle, J.J. Baumberg, and K. Köhler: Phys. Rev. Lett. 75, 2598 (1995).

39. N.H. Bonadeo et al: Science 282, 1473 (1998). 
40. M. Bayer et al: Science 291, 451 (2001).

41. B.E. Cole et al: Nature 410, 60 (2001).

42. G. Chen et al: Phys. Rev. Lett. 88, 117901 (2002).

43. X. Li et al: Science 301, 809 (2003).

44. D.P. DiVincenzo, Science 270, 255 (1995).

45. D. Mozyrsky, V. Privman, and M. L. Glasser: Phys. Rev. Lett. 86, 5112 (2001).

46. C. Piermarocchi et al: Phys. Rev. Lett. 89, 167402 (2002).

47. T. Calarco et al: Phys. Rev. A 68, 012310 (2003).

48. Optical Orientation, Modern Problems in Condensed Matter Science, Vol. 8, ed by F. Meier and B.P. Zakharchenya (North Holland, Amsterdam, 1984).

49. J.M. Kikkawa and D.D. Awschalom: Phys. Rev. Lett. 80, 4313 (1998); Phys. Today 52 (6), 33 (1999).

50. D. Gammon et al: Phys. Rev. Lett. 86, 5176 (2001).

51. Y. Kato et al: Nature (London) 427, 50 (2004).

52. D.P. DiVincenzo, Quantum computation and spin physics, J. Appl. Phys. 81 (1997), 4602-4607.

53. D.P. DiVincenzo, Quantum computing and single-qubit measurements using the spin-filter effect, J. Appl. Phys. 85 (1999), 4785-4787.

54. G. Burkard, D. Loss, and D.P. DiVincenzo, Phys. Rev. B 59, 2070 (1999).

55. X. Hu and S. Das Sarma, Phys. Rev. A 61, 062301 (2000).

56. M. Friesen et al: Phys. Rev. B 67, 121301 (2003).

57. B.E. Kane, N.S. McAlpine, A.S. Dzurak, R.G. Clark, G.J. Milburn, H.B. Sun, and H. Wiseman, Phys. Rev. B 61, 2961 (2000).

58. X. Hu, R. de Sousa, and D. Das Sarma: cond-mat/0108339. Published in the Proceedings of the 7th International Symposium on Foundations of Quantum Mechanics in the Light of New Technology (Tokyo, Japan, 2001).

59. C.P. Slichter: Principles of Magnetic Resonance, 3rd edn (Springer, Berlin 1990).

60. A. Abragam: Principles of Nuclear Magnetism (Oxford, Oxford 1961).

61. P. Meystre and M. Sargent III: Elements of Quantum Optics, 2nd edn (Springer-Verlag, Berlin 1991).

62. J. Fabian and S. Das Sarma: J. Vac. Sci. Technol. B 17, 1708 (1999).

63. L.A. Wu and D.A. Lidar: Phys. Rev. A 65, 042318 (2002).

64. L.A. Wu and D.A. Lidar: Phys. Rev. A 66, 062314 (2002).

65. A.V. Khaetskii and Yu.V. Nazarov: Phys. Rev. B 61, 12639 (2000); ibid. 64, 125316 (2001).

66. T. Fujisawa, Y. Tokura, and Y. Hirayama: Phys. Rev. B 63, 081304 (2001).

67. T. Fujisawa et al: Nature (London) 419, 278 (2002).

68. R. Hanson et al: Phys. Rev. Lett. 91, 196802 (2003).

69. M. Seck, M. Potemski, and P. Wyder: Phys. Rev. B 56, 7422 (1997); H.W. Jiang and E. Yablonovitch: Phys. Rev. B 64, R041307 (2001). See also, D. Stein, K. von Klitzing, and G. Weimann: Phys. Rev. Lett. 51, 130 (1983) for an early ESR measurement in GaAs heterostructures.

70. H.-A. Engel and D. Loss: Phys. Rev. Lett. 86, 4648 (2001).

71. D. Paget et al: Phys. Rev. B 15, 5780 (1977).

72. S.I. Erlingsson, Y.V. Nazarov, and V.I. Fal'ko: Phys. Rev. B 64, 195306 (2001).

73. R. de Sousa and S. Das Sarma: Phys. Rev. B 67, 033301 (2003); ibid. 68, 115322 (2003). 
74. G. Bastard: Wave mechanics applied to semiconductor heterostructures (Halsted, New York 1988).

75. J. Schliemann, D. Loss, and A.H. MacDonald, Phys. Rev. B 63, 085311 (2001).

76. X. Hu and S. Das Sarma: Phys. Rev. A 66, 012312 (2002).

77. K.V. Kavokin: Phys. Rev. B 64, 075305 (2001).

78. N.E. Bonesteel, D. Stepanenko, D.P. DiVincenzo: Phys. Rev. Lett. 87, 207901 (2001); G. Burkard and D. Loss: ibid 88, 047903 (2002); D. Stepanenko et al: Phys. Rev. B 68, 115306 (2003).

79. X. Hu and S. de Sousa, and S. Das Sarma: Phys. Rev. Lett. 86, 918 (2001).

80. X. Hu and S. Das Sarma: Phys. Rev. A 68, (2003).

81. J.Levy: Phys. Rev. Lett. 89, 147902 (2002).

82. D.P. DiVincenzo et al: Nature (London) 408, 339 (2000).

83. D.A. Lidar, I.L. Chuang, and K.B. Whaley: Phys. Rev. Lett. 81, 2594 (1998).

84. X. Hu and S. Das Sarma: Phys. Rev. A 64, 042312 (2001).

85. S. Vorojtsov, E.R. Mucciolo, and H.U. Baranger, cond-mat/0308118. To appear in Phys. Rev. B.

86. M. Friesen, R. Joynt, and M.A. Eriksson: Appl. Phys. Lett. 81, 4619 (2002).

87. W. Kohn, in Solid State Physics vol 5, page 257, ed. by F. Seitz and D. Turnbull (Academic, New York 1957).

88. K. Andres et al: Phys. Rev. B 24, 244 (1981).

89. B. Koiller, X. Hu, and S. Das Sarma: Phys. Rev. Lett. 88, 027903 (2002).

90. B. Koiller, X. Hu, and S. Das Sarma: Phys. Rev. B 66, 115201 (2002).

91. C.J. Wellard et al: Phys. Rev. B 68, 195209 (2003).

92. B. Koiller, R.B. Capaz, X. Hu, and S. Das Sarma: cond-mat/0402266.

93. T.B. Boykin et al: Appl. Phys. Lett. 84, 115 (2004).

94. A.A. Larionov, L.E. Fedichkin, and K.A. Valiev: Nanotechnology 12, 536 (2001).

95. A.J. Skinner, M.E. Davenport, and B.E. Kane, Phys. Rev. Lett. 90, 087901 (2003).

96. G.D.J. Smit et al: Phys. Rev. B 68, 193302 (2003).

97. A.S. Martins, R.B. Capaz, and B. Koiller: Phys. Rev. B 69, 085320 (2004).

98. D. Weinmann, W. Häusler, and B. Kramer: Phys. Rev. Lett. 74, 984 (1995).

99. M. Ciorga et al: Appl. Phys. Lett. 80, 2177 (2002).

100. K. Ono et al: Science 297, 1313 (2002).

101. A.K. Huttel et al: Europhys. Lett. 62, 712 (2003).

102. Single Charge Tunneling, ed. by H. Grabert and M.H. Devoret (Plenum, New York 1992).

103. R. Schoelkopf et al: Science 280, 1238 (1998).

104. M. Friesen et al: Phys. Rev. Lett. 92, 037901 (2004).

105. E. Pazy, T. Calarco, and P. Zoller: IEEE Trans. Nanotechnol. 3, 10 (2004).

106. G. Prinz: Phys. Today 45 (4), 58 (1995).

107. J.A. Sidles et al: Rev. Mod. Phys. 67, 249 (1995).

108. B. Koiller et al: Phys. Rev. Lett. 90, 067401 (2003).

109. L.P. Kouwenhoven, D.G. Austing, and S. Tarucha, Rep. Prog. Phys. 64, 701 (2001).

110. S. Das Sarma, J. Fabian, X. Hu, and I. Zutic: cond-mat/0002256, IEEE Trans. Magn. 36, 2821 (2000).

111. G.B. Lesovik, T. Martin, and G. Blatter: Euro. Phys. J. B 24, 287 (2001). 
112. N.M. Chtchelkatchev, G. Blatter, G.B. Lesovik, and T. Martin: Phys. Rev. B 66, 161320 (2002).

113. P. Recher, E.V. Sukhorukov, and D. Loss: Phys. Rev. B 63, 165314 (2001).

114. P. Recher and D. Loss: Phys. Rev. B 65, 165327 (2002).

115. C. Bena, S. Vishveshwara, L. Balents, and M.P.A. Fisher: Phys. Rev. Lett. 89, 037901 (2002).

116. R. Ionicioiu, P. Zanardi, and F. Rossi: Phys. Rev. A 63, 050101 (2001).

117. W. D. Oliver, F. Yamaguchi, and Y. Yamamoto: Phys. Rev. Lett. 88, 037901 (2002).

118. D.S. Saraga and D. Loss: Phys. Rev. Lett. 90, 166803 (2003).

119. X. Hu and S. Das Sarma: Phys. Rev. B 69, 115312 (2004).

120. P. Zhang, Q.K. Xue, X.G. Zhao, and X.C. Xie: cond-mat/0307037.

121. G. Burkard, D. Loss, and E. Sukhorukov: Phys. Rev. B 61, R16303 (2000).

122. S. Tarucha et al: Phys. Rev. Lett. 77, 3613 (1996).

123. M. Ciorga et al: Phys. Rev. B 61, R16315 (2000).

124. M. Pioro-Ladriere et al: Phys. Rev. Lett. 91, 026803 (2003).

125. J.M. Elzerman et al: Phys. Rev. B 67, 161308 (2003).

126. J.M. Elzerman et al: cond-mat/0312222.

127. F. Jelezko et al: Appl. Phys. Lett. 81, 2160 (2002).

128. H.J. Mamin et al: Phys. Rev. Lett. 91, 207604 (2003).

129. R. Budakian, H.J. Mamin, and D. Rugar: Phys. Rev. Lett. 92, 037205 (2004).

130. D. Gammon et al: Science 277, 85 (1997).

131. R.K. Kawakami et al: Science 294, 131 (2001).

132. K. Ono and S. Tarucha: cond-mat/0309062.

133. R.G. Clark et al: Phil. Trans. Roy. Soc. A 361, 1451 (2003).

134. Proceedings of the 1st International Conference on Experimental Implementation of Quantum omputation, ed. by R.G. Clark (Rinton, Princeton 2001).

135. M. Altarelli and W.L. Hsu, Phys. Rev. Lett. 43, 1346 (1979).

136. T. Shenkel et al: J. Vac. Sci. Technol B 20, 2819 (2002).

137. C.Y. Yang et al: Jpn. J. Appl. Phys. (part 1) 42 (6B), 4124 (2003).

138. J. L. O'Brien et al: Phys. Rev. B 64, 161401 (2001).

139. S. R. Schofield et al: Phys. Rev. Lett. 91, 136104 (2003).

140. V.W. Scarola And S. Das Sarma: cond-mat/0311463.

141. M. Stopa: unpublished. 


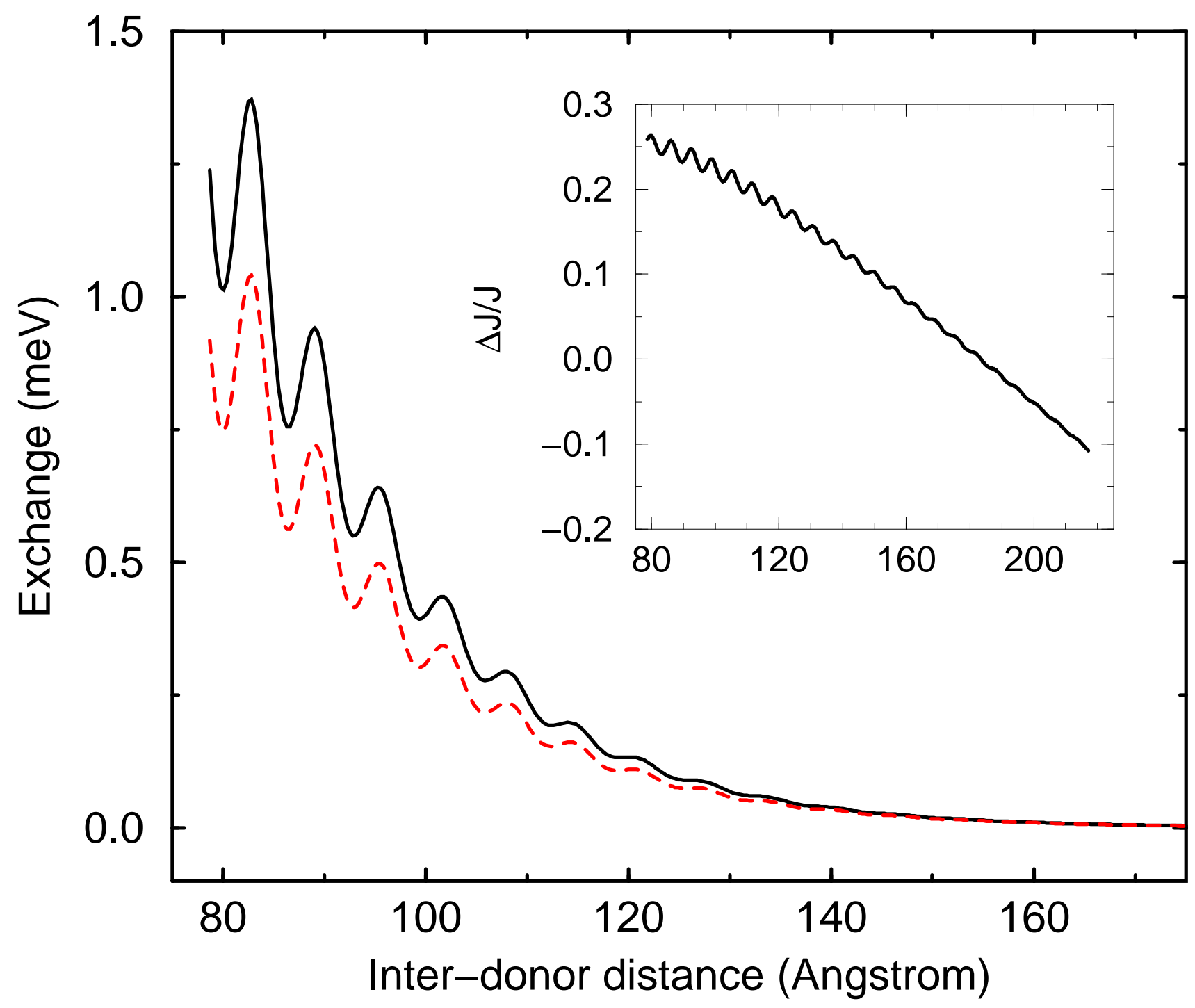

